\title{
NeuroImage
}

ELSEVIER

www.elsevier.com/locate/ynimg

NeuroImage 28 (2005) 154 - 164

\section{High gamma frequency oscillatory activity dissociates attention from intention in the human premotor cortex}

\author{
Andrea Brovelli, ${ }^{\mathrm{a}, *}$ Jean-Philippe Lachaux, ${ }^{\mathrm{b}}$ Philippe Kahane, ${ }^{\mathrm{c}}$ and Driss Boussaoud ${ }^{\mathrm{a}}$

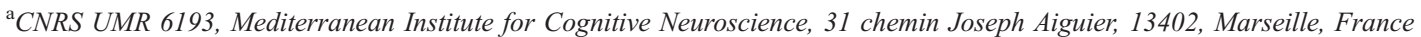 \\ b INSERM U280, Brain Signals and Processes Laboratory, 151 Cours Albert Thomas, 69003, Lyon, France \\ ${ }^{\mathrm{c}}$ Epilepsy Unit, CHU Grenoble, France
}

Received 21 December 2004; revised 17 March 2005; accepted 26 May 2005

Available online 14 July 2005

\begin{abstract}
The premotor cortex is well known for its role in motor planning. In addition, recent studies have shown that it is also involved in nonmotor functions such as attention and memory, a notion derived from both animal neurophysiology and human functional imaging. The present study is an attempt to bridge the gap between these experimental techniques in the human brain, using a task initially designed to dissociate attention from intention in the monkey, and recently adapted for a functional magnetic resonance imaging (fMRI) study [Simon, S.R., Meunier, M., Piettre, L., Berardi, A.M., Segebarth, C.M., Boussaoud, D. (2002). Task-related changes in cortical synchronization are spatially coincident with the hemodynamic response. Neuroimage, 16, 103-14]. Intracranial EEG was recorded from the cortical regions preferentially active in the spatial attention and/or working memory task and those involved in motor intention. The results show that, among the different intracranial EEG responses, only the high gamma frequency $(60-200 \mathrm{~Hz})$ oscillatory activity both dissociates attention/ memory from motor intention and spatially colocalizes with the fMRIidentified premotor substrates of these two functions. This finding provides electrophysiological confirmation that the human premotor cortex is involved in spatial attention and/or working memory. Additionally, it provides timely support to the idea that high gamma frequency oscillations are involved in the cascade of neural processes underlying the hemodynamic responses measured with fMRI [Logothetis, N.K., Pauls, J., Augath, M., Trinath, T. and Oeltermann, A. (2001). Neurophysiological investigation of the basis of the fMRI signal. Nature, 412, 150-7], and suggests a functional selectivity of the gamma oscillations that could be critical for future EEG investigations, whether experimental or clinical.

(C) 2005 Elsevier Inc. All rights reserved.
\end{abstract}

Keywords: Premotor cortex; Functional magnetic resonance imaging; Electrophysiology

\footnotetext{
* Corresponding author.

E-mail address: andrea.brovelli@incm.cnrs-mrs.fr (A. Brovelli).

Available online on ScienceDirect (www.sciencedirect.com).
}

\section{Introduction}

The primate dorsal premotor cortex (PMd) plays a major role in the selection, planning and execution of voluntary movements (for a review see Wise et al., 1997). Additionally, the PMd cortex is involved in nonmotor functions such as spatial attention and working memory (Boussaoud and Wise, 1993a,b; Corbetta et al., 1993; Coull and Nobre, 1998; Courtney et al., 1998). In fact, recent neurophysiological findings in monkeys suggest that attentional and mnemonic functions could be partially carried out by the rostral portion of PMd (PMdr), whereas motor preparatory processes would predominantly engage its caudal component (PMdc) (Boussaoud, 2001; Lebedev and Wise, 2001). Simon et al. (2002) tested this hypothesis in humans by means of a functional magnetic resonance imaging (fMRI) study and measured the brain activation triggered by an identical visual stimulus that could either direct spatial attention/working memory (SAM cue) or instruct motor preparation (motor instructional/conditional, MIC cue). The fMRI task was adapted from the experimental paradigm used to dissociate neuronal activity related to attention/memory from activity reflecting motor preparation in the monkey brain (Boussaoud and Wise, 1993a,b). Simon et al. (2002) developed two paradigms to cope with the slow hemodynamic response. In the attention/memory paradigm, the SAM stimulus was presented several times (up to 8 ) before the MIC cue was presented. This paradigm maximized attention/memory demands. In the motor preparation paradigm, the MIC cue was presented after a single SAM cue, and lasted for long and variable delays before a go signal told the subject to execute movement. Here, motor preparation was maximized. The results confirmed the organization found in the monkey and highlighted a differential recruitment of the PMd cortex together with the supplementary motor area (SMA) during motor preparation and spatial attention and memory (Simon et al., 2002).

However, a direct comparison with human fMRI studies is inevitably limited by the unresolved relation between functional 
imaging signals and basic neurophysiology. Simultaneous intracortical recordings of electrophysiological and hemodynamic responses are now starting to reveal which neural processes might trigger the cascade of changes in cerebral blood flow, volume and oxygenation, that form the basis for imaging studies such as fMRI. Although the blood oxygenation leveldependent (BOLD) response has been shown to correlate with the spiking activity averaged over an area of several millimeters (Kim et al., 2004), synaptic potentials seem to be the main cause of the hemodynamic response (Logothetis et al., 2001; Lauritzen and Gold, 2003; Logothetis and Wandell, 2004). The neuro-vascular relation can display both linear (Logothetis et al., 2001) and nonlinear (Lauritzen and Gold, 2003; Devor et al., 2003; Sheth et al., 2004) behaviors, and the stimulation paradigm (e.g., stimulus amplitude) seems to be one of the factors that can determine the type of coupling (Nemoto et al., 2004). Thus, these results suggest that imaging studies using subtraction-based analysis of hemodynamic signals produced by a complex experimental paradigm may not reflect corresponding differences in neural activations (Nemoto et al., 2004; Sheth et al., 2004).

This issue cannot be elucidated by simultaneous single-cell electrophysiological recordings and imaging techniques in humans for ethical reasons. However, intracerebral stereo-electroencephalography (SEEG) offers a valuable tool to measure integrated electrical phenomena at the millimeter scale (i.e., comparable to the fMRI spatial resolution) and a detailed analysis of its temporal dynamics could indirectly shed some light on some components of the neuro-vascular coupling in humans performing complex cognitive tasks. In fact, the SEEG signals clearly reveal the multidimensionality of the ensemble neuronal responses, which consist of event-related potentials (ERPs), induced synchronizations and desynchronizations in distinct frequency bands, whose relations with BOLD response remain elusive. In the present study, the local neural activity was recorded in an epileptic patient who had been stereotactically implanted with multicontact depth electrodes to monitor intractable epileptic seizures. The peculiarity of the implantation location within the frontal cortex (bilaterally) and the relative absence of epileptiform activities prompted us to perform a case study of this patient, while she performed a spatial attention and motor intention task similar to the one used in Simon et al. (2002). The aim of the study was to identify which SEEG oscillatory responses differentially engaged the human premotor cortex during spatial attention and/or memory vs. motor intention, and study those that mirrored previous fMRI interaction effects (Simon et al., 2002) so as to explore the relationship between electrophysiological and hemodynamic responses during a cognitive task.

\section{Materials and methods}

Patient, recordings and identification of SEEG sites within fMRI ROIs

The patient (a 40-year-old female) suffered from drug-resistant partial epilepsy and was a candidate for surgery. Since the location of the epileptic focus could not be identified using noninvasive methods, intracerebral recordings were performed by means of stereotactically implanted multilead depth electrodes. Fourteen semi-rigid electrodes were implanted in cortical areas adapted to the suspected origin of seizures. The selection of implantation sites was dictated solely by clinical aspects with no reference to the present experimental protocol; however, the patient was selected because her implantation sampled a frontal network which is thought to be involved in selective visual attention and motor intention in humans (Simon et al., 2002). The patient performed the task 4 days after the implantation of the electrodes and had previously given her informed consent to participate in the experiment. Each electrode had a diameter of $0.8 \mathrm{~mm}$ and comprised from 7 to 15 leads, $2 \mathrm{~mm}$ in length and $1.5 \mathrm{~mm}$ apart (that is, $3.5 \mathrm{~mm}$ center to center) (Dixi, Besançon, France). The electrode contacts were identified on each individual stereotactic scheme, and then anatomically localized using the proportional atlas of Talairach and Tournoux. In addition, the computer-assisted matching of postimplantation CT-scan with a pre-implantation 3-D MRI (VOXIM R, IVS Solutions, Germany) provided a direct visualization of the electrode contacts with respect to the brain anatomy of the patient. A total of 30 bipolar derivations were identified within the frontal cortical surface. The Talairach coordinates of four fMRI Regions Of Interest (ROIs) preferentially active for spatial attention/memory (referred to as SAM in Table 1) or motor preparation (MIC 1, MIC 2 and MIC 3 in Table 1) within the frontal cortex were taken as reference points from Simon et al. (2002, Interaction analysis, Table 4). The SAM region of interest was located in the right PMd cortex, MIC 1 and 2 were on the cingulate gyrus and supplementary motor area, whereas MIC 3 laid in the left PMd cortex. The distance between the ROIs and each bipolar derivation was computed so as to determine the relevance of each SEEG response on the basis of its proximity to the fMRI ROIs.

\section{Behavioral paradigm}

The task design is similar to the one used in previous electrophysiological studies in monkeys (Boussaoud and Wise, 1993a,b). The subject was asked to perform a conditional visuomotor task. Visual cues were shown on a video monitor placed $70 \mathrm{~cm}$ from the subject, and motor responses consisted of pressing one of two mouse buttons. Because most of the electrodes were implanted in the right hemisphere for clinical reasons, the subject was asked to perform the task with her left hand. A trial begins with the presentation of a fixation circle $\left(\sim 1^{\circ}\right.$ diameter) at the center of the monitor, and the subject had to move her gaze to the circle and maintain fixation throughout the trial. After a fixation delay of $0.8,1$ or $1.2 \mathrm{~s}$, a white square $\left(1.8^{\circ} \times 1.8^{\circ}\right)$ appeared at one of four locations (see Fig. 1a), with an eccentricity of $6.6^{\circ}$. This first stimulus is termed spatial attentional and/or mnemonic (SAM) cue (Boussaoud and Wise, 1993a,b); it was presented for $250 \mathrm{~ms}$. After a delay of $0.8,1$ or $1.2 \mathrm{~s}$ following the SAM cue offset, a motor instructional conditional (MIC) cue appeared. In $75 \%$ of the trials, the MIC cue consisted of a single square (either red or green) presented at the same location as the SAM cue. In $25 \%$ of the trials, the MIC cue was composed of two squares (one red and one green) presented simultaneously, one at the same location as the SAM cue, the other at a nearby location. The response rule required the subject to select the square at the SAM cue's location, discriminate its color and press the left or the right button of the mouse, placed underneath the middle and index fingers, according to a conditional rule: red meant to press with the 
Table 1

Summary of the spectral analysis in relation with fMRI regions of interest (ROI) ${ }^{\mathrm{a}}$

\begin{tabular}{|c|c|c|c|c|c|c|c|c|c|}
\hline \multicolumn{2}{|l|}{ SEEG } & \multicolumn{4}{|c|}{ Distance from fMRI ROIs } & \multicolumn{2}{|c|}{ Left stimulus } & \multicolumn{2}{|c|}{ Right stimulus } \\
\hline Bipolar name & Site \# & SAM & MIC 1 & MIC 2 & MIC 3 & $\Delta t(\mathrm{~ms})$ & $\Delta f(\mathrm{~Hz})$ & $\Delta t(\mathrm{~ms})$ & $\Delta f(\mathrm{~Hz})$ \\
\hline V8-9 & 1 & 8 & 32 & 24 & 69 & $220-280$ & $120-160$ & ns & ns \\
\hline V11-12 & 2 & 12 & 43 & 34 & 80 & $\begin{array}{l}0-200 \\
200-400 \\
380-440\end{array}$ & $\begin{array}{l}9-13 \\
13-21 \\
70-110\end{array}$ & ns & ns \\
\hline A $8-9$ & 3 & 15 & 74 & 38 & 27 & ns & ns & $\mathrm{ns}$ & ns \\
\hline $\mathrm{Y} 3-4$ & 4 & 28 & 17 & 2 & 49 & $\begin{array}{l}0-300 \\
280-320\end{array}$ & $\begin{array}{l}13-29 \\
80-110\end{array}$ & $\begin{array}{l}0-400 \\
\text { ns }\end{array}$ & $\begin{array}{l}17-29 \\
\text { ns }\end{array}$ \\
\hline$V^{\prime} 2-3$ & 5 & 40 & 12 & 16 & 35 & $\begin{array}{l}200-400 \\
240-480\end{array}$ & $\begin{array}{l}21-29 \\
100-200\end{array}$ & $\begin{array}{l}200-350 \\
380-500 \\
300-460\end{array}$ & $\begin{array}{l}21-29 \\
70-100 \\
110-200\end{array}$ \\
\hline$Y 7-8$ & 6 & 33 & 28 & 13 & 56 & ns & ns & ns & ns \\
\hline $\mathrm{A} 1-2$ & 7 & 29 & 18 & 6 & 48 & ns & ns & $\mathrm{ns}$ & ns \\
\hline $\mathrm{V} 1-2$ & 8 & 30 & 9 & 11 & 43 & ns & ns & ns & ns \\
\hline $\mathrm{V}^{\prime} 6-7$ & 9 & 56 & 23 & 33 & 22 & $10-140$ & $110-160$ & $\begin{array}{l}0-140 \\
0-140\end{array}$ & $\begin{array}{l}130-200 \\
60-100\end{array}$ \\
\hline Q6-5 & 10 & 56 & 73 & 64 & 107 & $620-700$ & $16-22$ & ns & ns \\
\hline$V^{\prime} 12-13$ & 11 & 74 & 42 & 51 & 17 & $50-350$ & $16-23$ & ns & ns \\
\hline B9-10 & 12 & 23 & 48 & 39 & 84 & $50-200$ & $13-25$ & $80-350$ & $9-25$ \\
\hline
\end{tabular}

${ }^{a}$ The first and second columns from the left list the SEEG bipolar recording names and site number. Columns 3 to 6 display the distance in millimeters between each SEEG bipolar recording and the fMRI ROIs. Columns 7 and 8 show the time $(\Delta t)$ and frequency $(\Delta f)$ intervals, which displayed a significant difference in mean power after a left hemifield SAM and MIC cue. Columns 9 and 10 show the time and frequency intervals of significant difference after a right hemifield SAM and MIC cue (ns, not significant).

middle finger, green with the index finger. The MIC cue lasted for a variable delay period $(0.8,1$ or $1.2 \mathrm{~s})$, and its offset served as the GO signal. Different types of trials were presented in a random order and the subject could not anticipate the location of the upcoming SAM cue, nor the color or number of the MIC cues. In this situation, when the SAM cue was followed by a single square as the instructional cue ( $75 \%$ of the trials), the same visual stimulus guided spatial attention and/or memory during the first part of the trial and instructed a movement during the second part of the same trial. The rationale for the $25 \%$ double MIC cues trials was to force the subject to focus her attention on the SAM cue location. Indeed, in this type of trials, the subject would perform at chance if she did not use the attentional cue. Thus, only the one MIC cue trial was selected and further analyzed. Any SEEG response found to be higher following the SAM cue than after the MIC cue is considered as preferentially related to spatial attention and/or memory. In the opposite case, the SEEG response is considered to reflect mostly motor preparation.

\section{Data analysis}

The experiment consisted of 300 trials, and the number of trials with the SAM and MIC cue at each of the four locations was of equal number. Trials with cue locations in the same visual hemifield were grouped and trials containing early responses (i.e., finger movements before the GO cue) and incorrect responses were eliminated. The limited number of early and incorrect trials did not allow us to analyze them separately. This gave a total of 201 trials with a single SAM and MIC cue (101 trials for the left hemifield cue and 100 for the right hemifield cue). Thus, 4 groups of SEEG responses (conditions) were created: two for the SAM cue (left and right hemifield) and two for the MIC cue (left and right hemifield).
Our analysis searched for SEEG responses comparable in spatial resolution with the fMRI regions of interest (ROIs) found in a previous study (Simon et al., 2002). Therefore, bipolar derivations were computed between adjacent electrode contacts to suppress contributions from nonlocal assemblies and assure that the bipolar SEEG signals could be considered as originating from a cortical volume centered within two contacts (the intra-contact distance was $1.5 \mathrm{~mm}$ ). In fact, the spatial resolution of such bipolar recordings has been estimated as being around $4 \mathrm{~mm}$ (Lachaux et al., 2003), which is comparable with the standard fMRI voxel size. We carefully looked for any sign of epileptiform activity, but none was found in any of the bipolar derivations during the experiment. Average event-related potentials (ERPs) were computed for each bipolar derivation and condition. Secondly, we used spectral analysis based on continuous wavelet transforms to characterize the time-frequency structure of the bipolar SEEG signals (Tallon-Baudry and Bertrand, 1999). The time-frequency (TF) power representations of the bipolar SEEG were computed for each single trial and then averaged for each experimental condition. TF analysis was performed by convolution with complex gaussian Morlet's wavelets with a $f / \sigma_{f}$ ratio of 10 , where $f$ is the central frequency of the wavelet and $\sigma_{f}$ is its standard deviation in frequency. The central frequency varied from 2 to $200 \mathrm{~Hz}$, in step of $2 \mathrm{~Hz}$. Since each wavelet family is characterized by a fixed relation between the standard deviation in frequency $\sigma_{f}$ and time $\sigma_{t}\left(\sigma_{f}=1 /\left(2 \pi \sigma_{t}\right)\right.$ for the Morlet's wavelets), the wavelet resolution depends exclusively on the central frequency $f$. This leads to a wavelet with a resolution of $148 \mathrm{~ms}$ and $4 \mathrm{~Hz}$ at $20 \mathrm{~Hz}$, and of $18 \mathrm{~ms}$ and $36 \mathrm{~Hz}$ at $180 \mathrm{~Hz}$. We used a nonparametric Kruskal-Wallis test for all statistical comparisons. This test was applied to find the significant differences in mean evoked potentials and mean TF power values across conditions. All four pairs of conditions were compared (left MIC cue vs. left SAM cue, right MIC cue vs. 
a

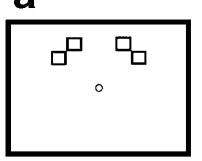

b

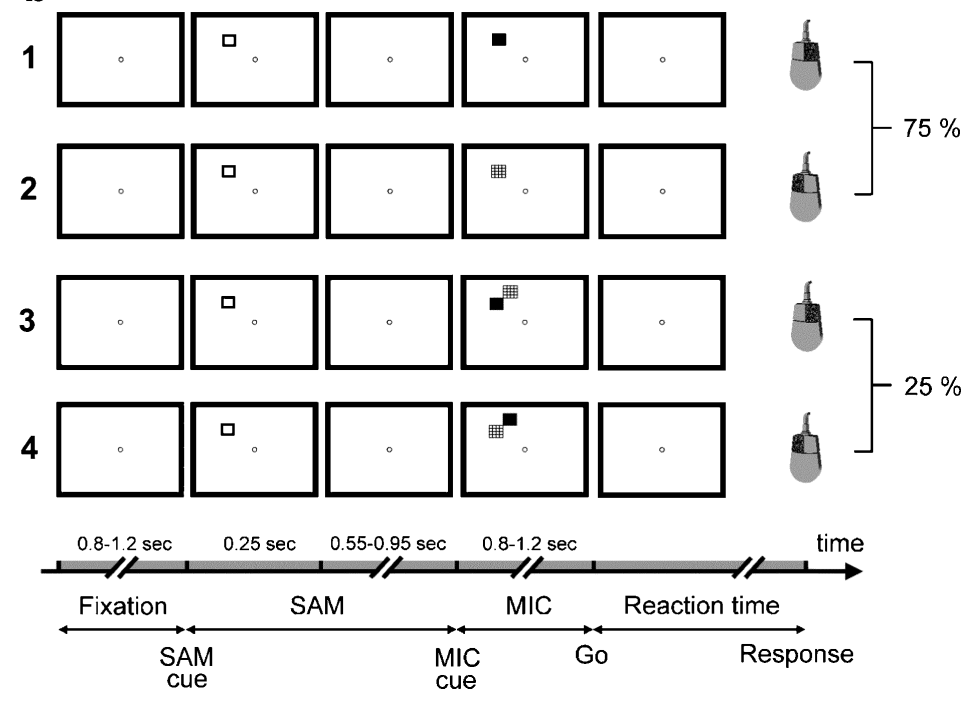

Fig. 1. Experimental paradigm: examples of trials and temporal events. (a) Diagram showing the location of the fixation point (central circle) and the four possible locations of visual cue appearance (squares). (b) The rectangles depict the state of the video screen at the major steps of the task during 4 representative trials (1-4). Each trial began with a fixation period of varying duration (Fixation) followed by the appearance of a stimulus at one of the four locations termed spatial attentional and/or mnemonic (SAM) cue, presented for $250 \mathrm{~ms}$. After a delay of $0.8,1$ or $1.2 \mathrm{~s}$ following the SAM cue offset, a motor instructional conditional (MIC) cue appeared. In 75\% of the trials, the MIC cue consisted of a single square (either red or green, here drawn as dark and light grey) presented at the same location as the SAM cue (trials 1 and 2). In $25 \%$ of the trials, the MIC cue was composed of both a red and a green square, one of which was at the same location as the previous SAM cue (trials 3 and 4). The response rule required the subject to select the square at the SAM cue's location, discriminate its color and press a mouse button according to a conditional rule: red meant to press the right button of a mouse (left) with the middle finger, green meant to press the left button with the index finger.

right SAM cue, left MIC cue vs. right MIC cue, left SAM cue vs. right SAM cue). To compare the ERPs between the two conditions of a pair, we used a sliding window of $50 \mathrm{~ms}$ duration regularly shifted in $25 \mathrm{~ms}$ steps so as to cover a time interval ranging from 0 to $800 \mathrm{~ms}$ after cue onset. For each step, the test compared the average value of the ERPs during the 50 ms window. To compare mean TF power values, the TF maps were divided into a set of overlapping time-frequency tiles. A first tiling used $[150 \mathrm{~ms} \times 8 \mathrm{~Hz}]$ windows with $[50 \mathrm{~ms} \times 4 \mathrm{~Hz}]$ steps to cover frequencies ranging from 2 to $60 \mathrm{~Hz}$; for higher frequencies, we used a second tiling with [60 $\mathrm{ms} \times 30 \mathrm{~Hz}]$ windows with a $[20 \mathrm{~ms} \times 10 \mathrm{~Hz}] \mathrm{step}$, it covered the [30 to 200 $\mathrm{Hz}$ ] frequency range. The use of two different meshes for low and high frequencies was decided to adapt the statistical test to the varying temporal and frequency resolution of the wavelets. In order to avoid spurious positives and to take into account the large number of comparisons tested, the significance level of 0.01 was lowered to account for the number of comparisons being performed using the Bonferroni method. Thus, the significance level of 0.01 was divided by the number of SEEG sites (30) and by the number of windows ( 32 for the ERP, 196 for the low frequency range and 570 for the high frequency range). The corrected level of significance was then $P<1.04 \times 10^{-5}$ (e.g., $P<0.01 / 30 / 32), 1.7 \times 10^{-6}$ and $5.85 \times 10^{-7}$, for the ERP, low frequency and high frequency range, respectively. Then, in order to evaluate the sign of the difference between conditions, the mean power waveforms were transformed to $z$-scores with respect to prestimulus activity (from $1 \mathrm{~s}$ to $0.3 \mathrm{~s}$ before fixation onset). To allow direct comparison, the same baseline was taken for both the SAM and MIC SEEG responses. SEEG signals were evaluated with the software package for electrophysio- logical analysis (ELAN-Pack) developed in the INSERM U280 laboratory.

\section{Results}

This paper focuses on the SEEG responses (i.e., the eventrelated potentials and the mean power changes in different frequency bands) that differentiate spatial attention/working memory from motor preparation in the human premotor cortex, and on their correspondence with the BOLD effects observed by Simon et al. (2002). Thus, we first checked the location of the SEEG sites with respect to the fMRI ROIs. Eight of the 30 bipolar derivations were located relatively closely (less than 15 $\mathrm{mm}$ ) to the center of at least one of the four fMRI regions of interest defined within the frontal cortex (SEEG sites from 1 to 8 in Table 1, Fig. 2). Unfortunately, no SEEG derivations were close to the third MIC related ROI (MIC 3). Thus, except for MIC 3, we were able to measure the electrical activity of cortical sites that displayed significant changes in hemodynamic response to the SAM and MIC cues and study their dynamics of activation (Fig. 2).

\section{ERPs and fMRI ROIs}

The statistical comparison between the mean ERPs triggered by the SAM and MIC cues (from 0 to $800 \mathrm{~ms}$ after cue onset) revealed significant differences in 21 and 18 bipolar derivations for the left and right hemifield cue, respectively. These included the 8 locations close to the fMRI ROIs, but spanned a broader cortical area of the frontal cortex (Fig. 3). Since, there was no 

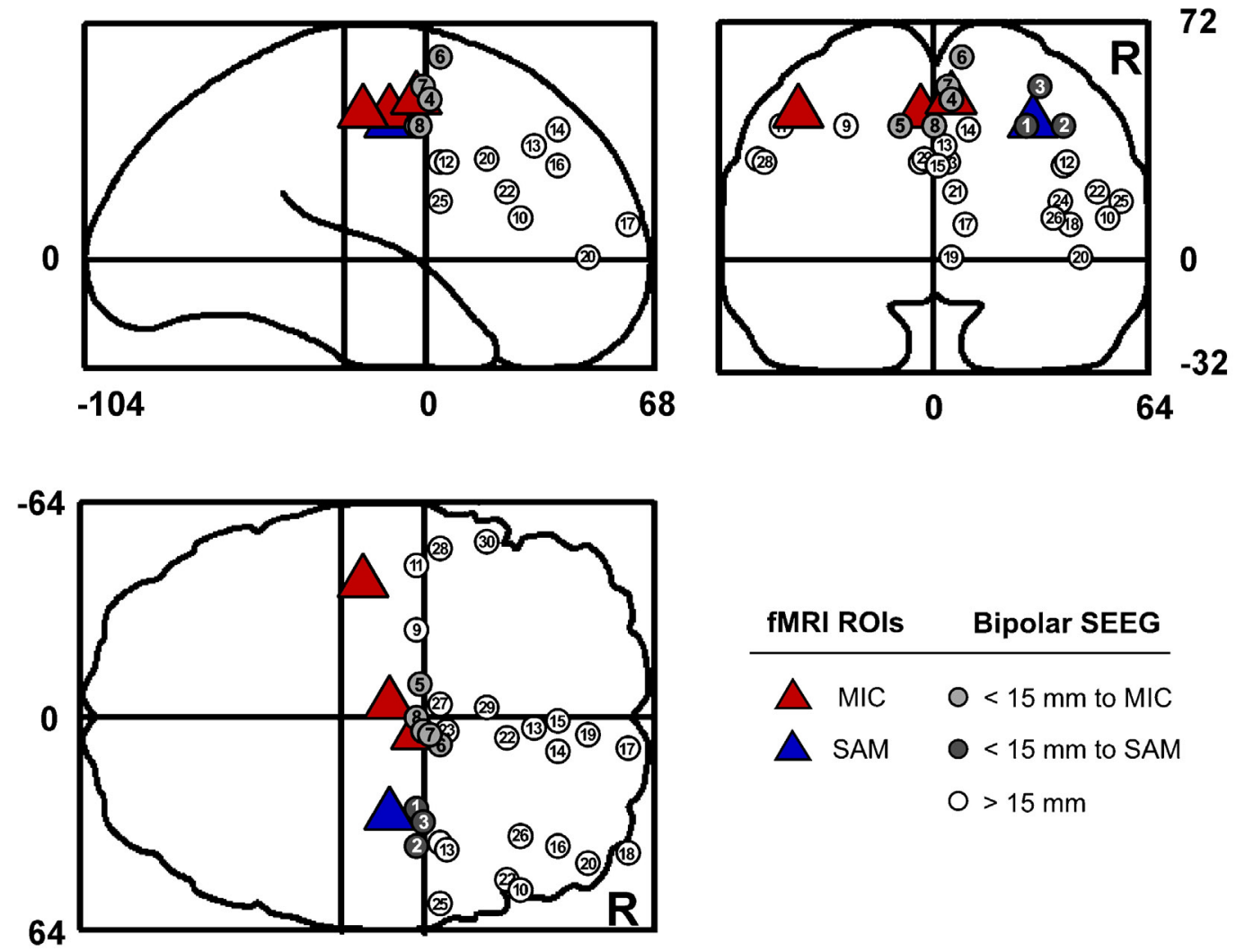

Fig. 2. Location of the SEEG sites and fMRI regions of interest (ROIs). The SEEG electrode contacts were identified on each individual stereotactic scheme, and then anatomically localized using the proportional atlas of Talairach and Tournoux. Thirty SEEG bipolar derivations were selected for analysis and are represented as numbered circles 1 to 30. Eight bipolar SEEG derivations (numbered from 1 to 8 ) were in proximity (i.e., $<15 \mathrm{~mm}$ ) to at least one of the two fMRI ROIs. SEEG sites closely placed to the SAM and MIC ROIs are coded with different shades of gray. The SAM and the MIC ROIs are coded as blue and red triangles, respectively. The Talairach coordinates of the fMRI ROIs are $(29,-11,42)$ for SAM, $(-4,-11,41)$ for MIC1, $(5,-3,49)$ for MIC2 and $(-40$, $-19,45)$ for MIC3 (see also Table 1).

spatial correspondence between the ERP variations and fMRI data, we did not pursue a more detailed analysis of the average ERP.

\section{Beta band responses and fMRI ROIs}

A nonparametric statistical analysis was performed to assess whether the time-frequency (TF) mean power maps of the bipolar SEEG signals differed between the SAM and MIC periods of the task (Fig. 1) in the low frequency band $(2-60 \mathrm{~Hz})$. Comparisons were performed independently for the left and right hemifield cues, and a significant difference (corrected $P<0.01$ ) between the SEEG responses after the SAM and MIC cues was found in the beta frequency band $(15-30 \mathrm{~Hz})$. These differences were observed in sites 2, 4, 5, 10, 11 and 12 for left hemifield cues, and in sites 4, 5 and 12 for the right hemifield cues (Table 1). Among those sites, one SEEG site (site 2) was located within $15 \mathrm{~mm}$ from the second SAM-related fMRI ROI and two sites (site 4 and 5) were closely placed to the MIC-related fMRI ROI (Fig. 2). However, the effects observed in the beta range did not match those observed in the fMRI study. In fact, all three sites displayed a significant decrease in beta band power after the MIC cue with respect to the SAM cue, independently of the distance to different fMRI ROIs (Fig. 4). The other sites with a significant difference in the beta band (sites 10,
11 and 12) were located 23 to $107 \mathrm{~mm}$ from any of the fMRI ROIs. The remaining five SEEG sites (sites 1, 3, 6, 7 and 8) that were located close to the fMRI ROIs did not display significant differences in the beta-band mean power between conditions. Thus, despite significant differences between SAM and MIC cues in the beta band SEEG responses, there was a limited degree of overlap with fMRI ROIs. No further analysis was performed in this frequency band.

\section{Gamma band responses and fMRI ROIs}

The analysis was then extended to the high frequency band ranging from 30 to $200 \mathrm{~Hz}$. As in the beta band, significant differences in mean power between the SAM and MIC conditions were found in the gamma band (see Table 1). Most of the differences were found in a time interval from approximately 200 to $450 \mathrm{~ms}$ and in a frequency band ranging from 60 to $200 \mathrm{~Hz}$ (Table 1, columns 7 to 10). The significant effect had an average duration of $70 \mathrm{~ms}$ spread over a frequency band of $50 \mathrm{~Hz}$ for the left cues, and $140 \mathrm{~ms}$ and $60 \mathrm{~Hz}$ for the right cues. For the left cues, five sites (sites 1, 2, 4, 5 and 9) showed a significant difference in mean power and 4 of them were located less than $15 \mathrm{~mm}$ from the fMRI ROIs (Fig. 4). Most importantly, sites 1 and 2, whose activity after the SAM cue was significantly higher 

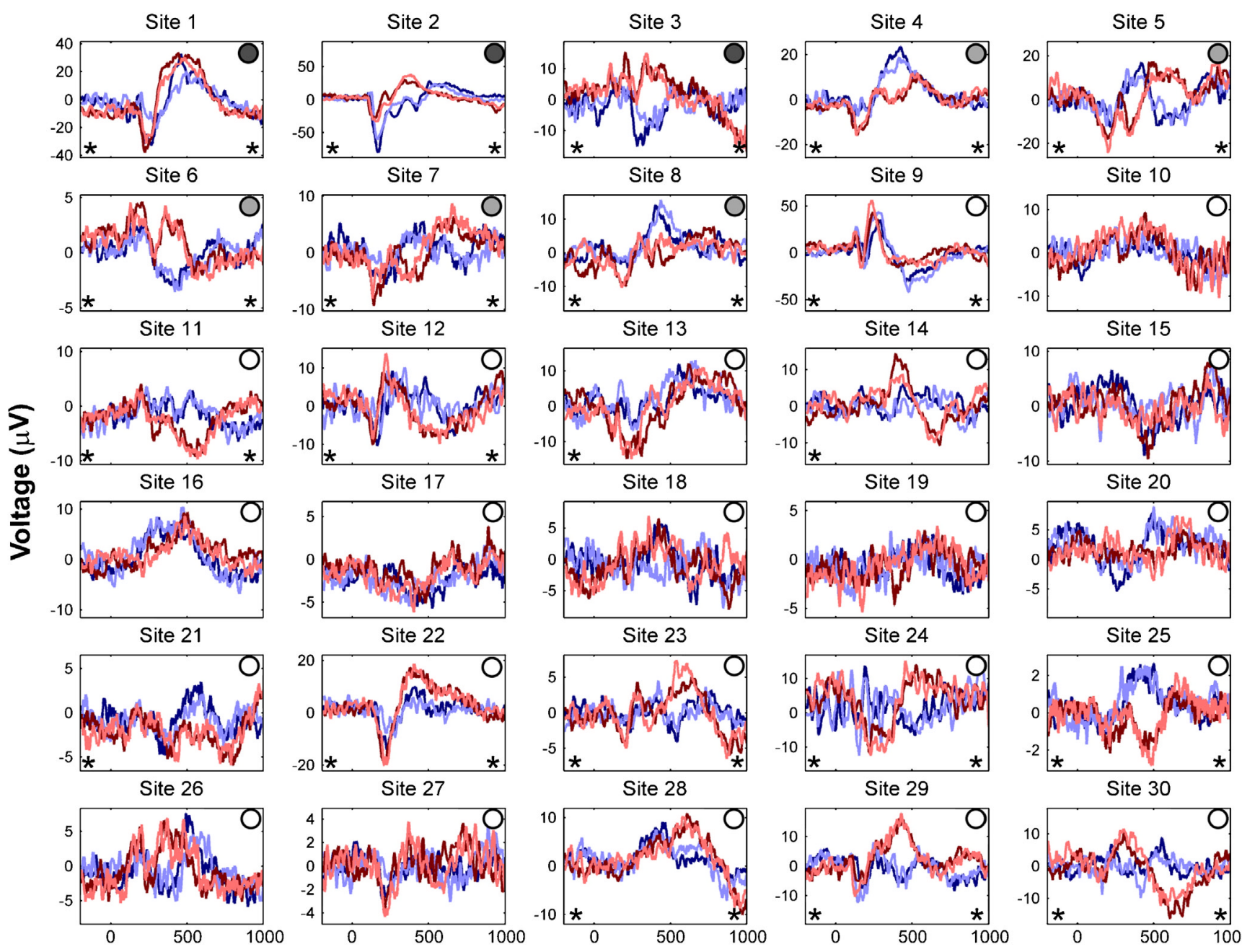

Site 24
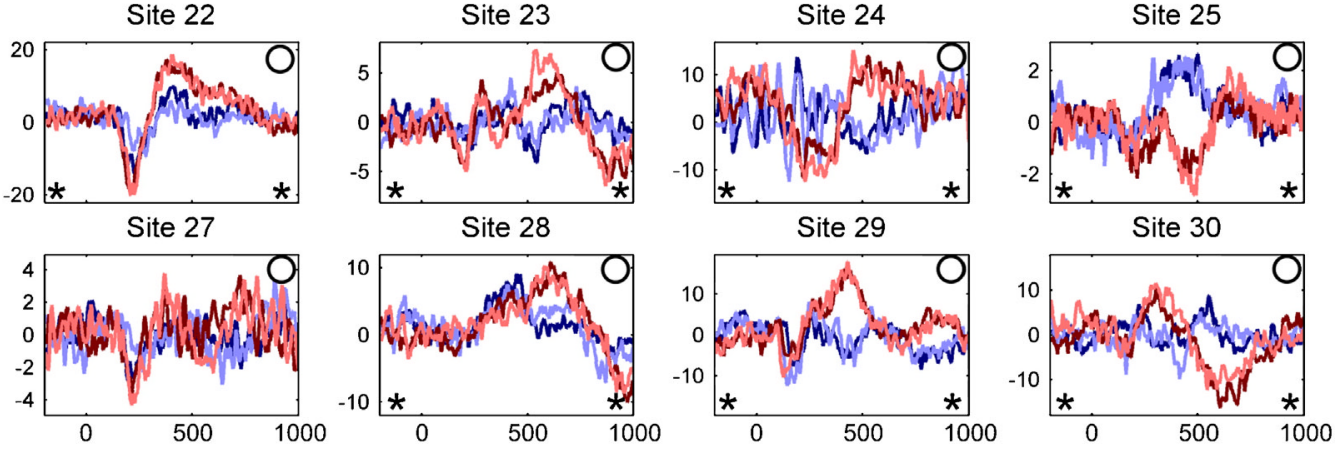

Time (msec)

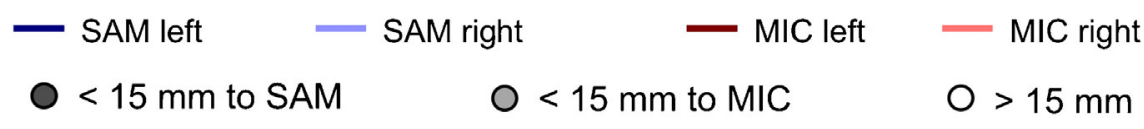

* significant difference between SAM and MIC ERPS

\section{Legend}

Fig. 3. Time course of the SAM and MIC ERPs. The ERPs for each SEEG site (sites 1 to 30) are coded with different colors (see legend). A circle in the upperright corner of each panel indicates the distance of each site from the fMRI ROIs. The asterisk in each panel indicates whether the corresponding SAM and MIC ERPs differ significantly at least in one $50 \mathrm{~ms}$ window between 0 and $800 \mathrm{~ms}$ after stimulus onset. The location of the asterisk, bottom-left and/or bottomright corners, indicates whether the difference between SAM and MIC was for the left and/or the right hemifield stimulation.

than after the MIC cue (Fig. 5a), were located 8 and $12 \mathrm{~mm}$ from the ROI preferentially active after SAM cue (Table 1 , columns 3 to 5, Fig. 6), within the dorsal premotor cortex PMd. In a symmetrical effect, sites 4 and 5 were preferentially active after the MIC cue and were located 2 and $12 \mathrm{~mm}$ from the fMRI ROI significantly more active during the MIC condition (i.e., ROIs MIC 1 and 2; Fig. 5b, left panel). These sites were located within the medial bank of the PMd cortex and in the cingulate motor area (CMA), respectively. For the right cue comparison, 2 sites (site 5 and 9) displayed significant differences in mean power between right SAM and MIC cues. Site 5 was selectively active for the MIC cue and it was located $12 \mathrm{~mm}$ from MIC 1 (Fig. 6).
Also, site 9 displayed a selectivity for the MIC cue, but it was not located close to any fMRI ROI (Fig. 5b, right panel). Finally, four SEEG sites (sites 3, 6, 7 and 8) that were closely placed to the fMRI ROIs did not display significant difference in SEEG mean power response.

\section{Temporal dynamics of SEEG response in the gamma band}

The temporal dynamics of the SAM selective sites (sites 1 and 2) were characterized by a steep increase in SEEG response $150 \mathrm{~ms}$ after cue onset, followed by a slower decay and a return to the baseline level around $600 \mathrm{~ms}$ thereafter (Fig. 5a). On the 
a

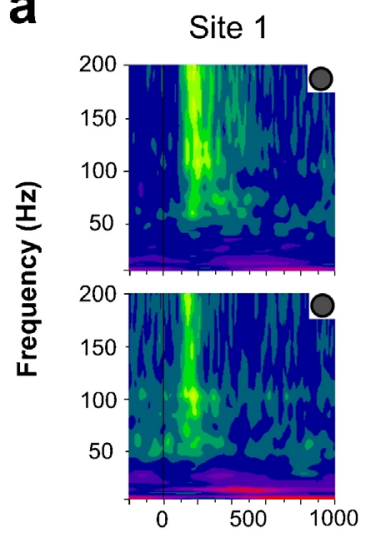

Site 9

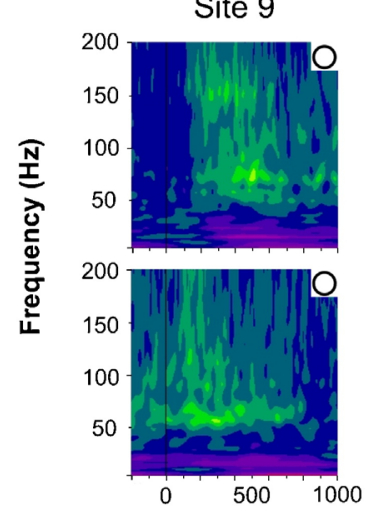

b

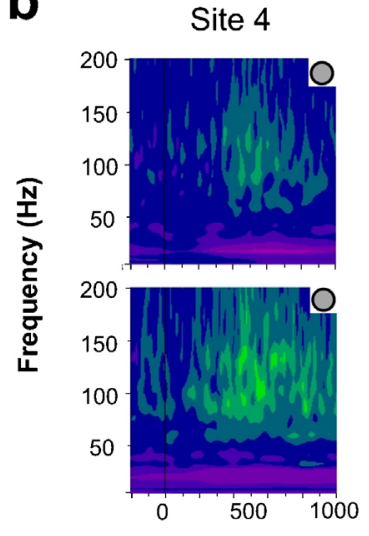

Site 2
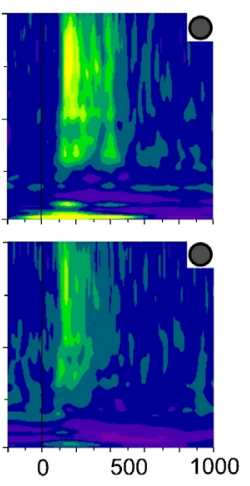

Site 10

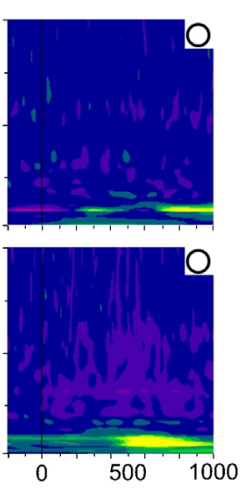

Site 4
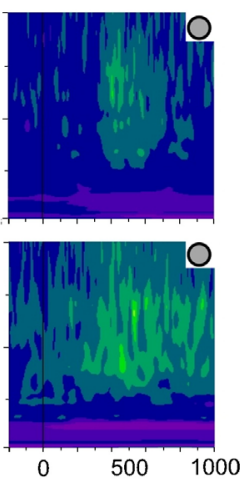

Site 11
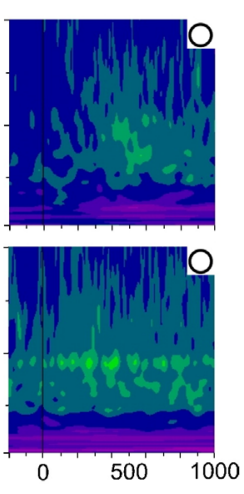

Time (ms)

Site 5
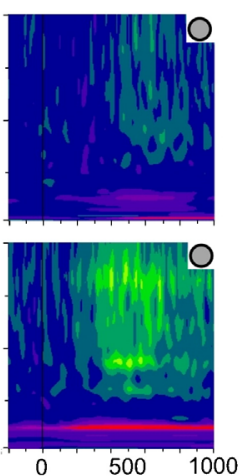

Site 9
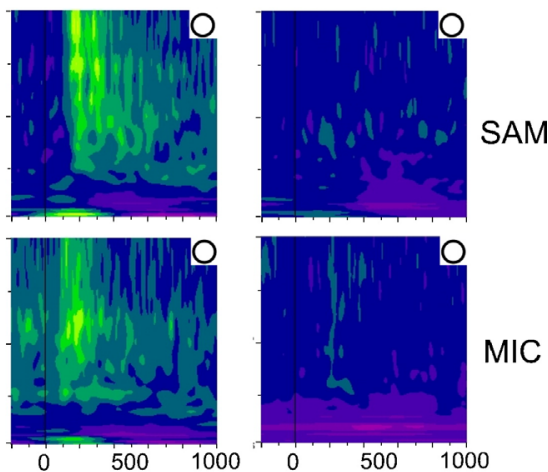

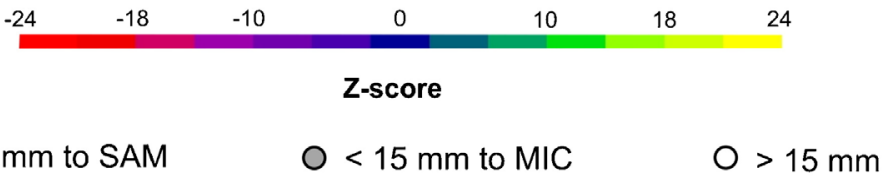

Legend

Fig. 4. Time-frequency maps of the SEEG sites showing significant difference in mean power. (a) Time-frequency maps of the SEEG sites displaying a significant difference in either the beta and/or high gamma band to the left hemifield SAM cue (top panel for each site) over the left hemifield MIC cue (bottom panel for each site). (b) Time-frequency maps of the SEEG sites displaying a significant difference in either the beta and/or high gamma band to the right hemifield SAM cue over the right hemifield MIC cue. A circle in the upper-right corner of each panel informs about the distance of each site from the fMRI ROIs (see legend). 
a

\section{Left}

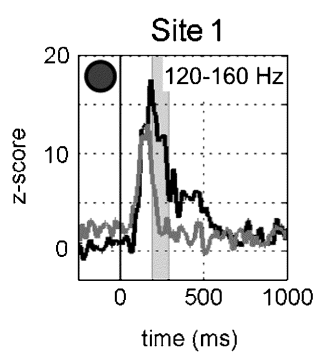

Site 2

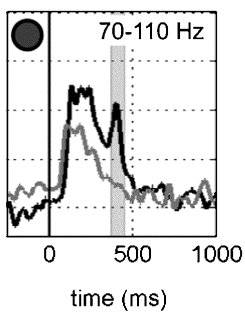

\section{Legend}

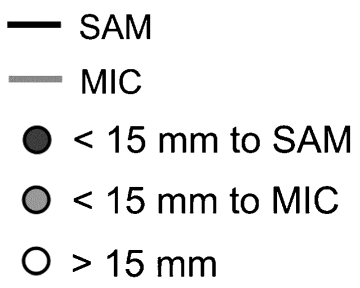

b

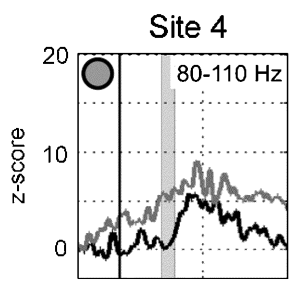

Site 9

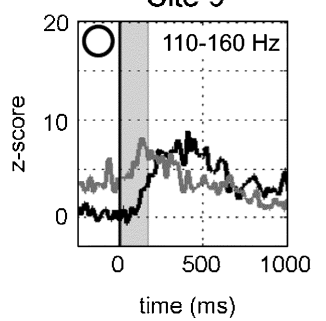

Left

Site 5

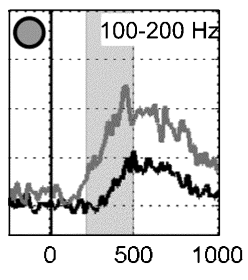

time (ms)

\section{Right}

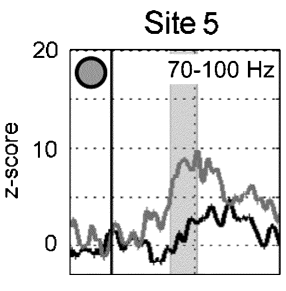

Site 9

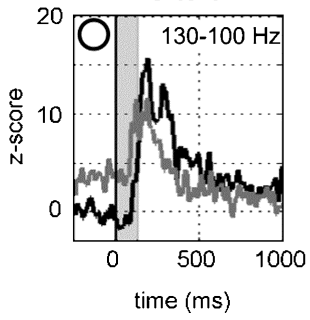

Site 5

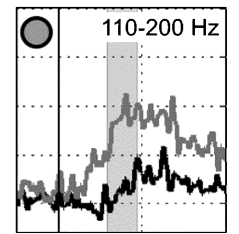

Site 9

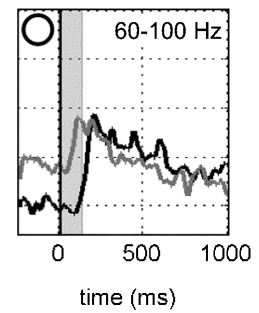

Fig. 5. Time course of high gamma frequency oscillations. The mean power waveforms were transformed to $z$-scores with respect to the same baseline interval (fixation period) and averaged over the frequency range displaying significant difference across conditions (top right inset in each panel, taken from Table 1). The time interval of significant difference across conditions is defined by the shaded area. (a) High gamma frequency time course in cortical sites displaying a preferential response to the left hemifield SAM cue (black line) over left hemifield MIC cue (grey line). (b) Time course of sites selective for the MIC cue. The three left panels show the time courses after a left hemifield cue, whereas the four panels on the right refer to a right hemifield cue.

other hand, the MIC selective sites (sizes 4 and 5) were characterized by a relatively slower increase in SEEG response starting approximately at $200 \mathrm{~ms}$, peaking around $500 \mathrm{~ms}$ and remaining sustained until the GO signal (from 0.8 to $1.2 \mathrm{sec}$; Fig. 5 b, left panel). Finally, site 9, which was located away from the MIC related ROIs, showed a stronger increase after the MIC cue with a mixed dynamics composed of a fast onset and a sustained response until the GO signal.

\section{Discussion}

The present study aimed at identifying components of the electrophysiological activity in the human premotor cortex that might dissociate spatial attention/memory from motor planning in a way that would mirror our previous fMRI results. As stated in the Introduction, the task used in the fMRI study and the present one address the same neural processes. We found that (1) ERPs as well as the beta frequency $(15-30 \mathrm{~Hz})$ of the SEEG activity, differentiate between attentional/mnemonic cues, but have weak or no spatial relation with the BOLD response measured in a previous study using the same task in terms of processing (Simon et al., 2002); (2) the high gamma frequency $(60-200 \mathrm{~Hz})$ of the SEEG activity both reflects attentional/mnemonic vs. motor intention processes and colocalizes with fMRI regions of interest. We will discuss these findings in light of previous studies that attempted to determine the neurophysiological correlate of the BOLD signal.

ERPS and SEEG in the beta frequency do not colocalize with the BOLD signal

The analysis of the mean ERPs triggered by the SAM and MIC cues revealed significant differences in most cortical SEEG sites, spanning the whole frontal cortex. This result apparently contradicts the previous fMRI study (Simon et al., 2002) which showed that discrete regions of the premotor cortex dissociated between spatial attention/working memory and motor planning. Indeed, the difference in ERP extended to a broader cortical region than the ones outlined by the fMRI study. This result is in 


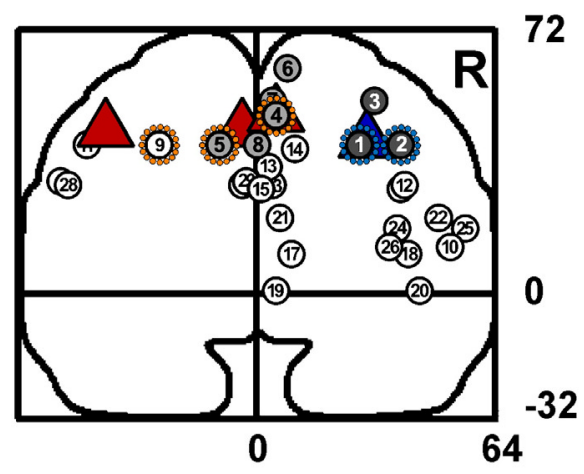

fMRI ROls Bipolar SEEG

$\begin{array}{ll}\triangle \text { MIC } & 0<15 \mathrm{~mm} \text { to MIC } \\ \triangle \text { SAM } & 0<15 \mathrm{~mm} \text { to SAM } \\ & 0>15 \mathrm{~mm}\end{array}$

\section{SEEG high-gamma band effect}

$\begin{array}{ll}8_{0000}^{8000} \\ 8 & M I C>S A M \\ 8000 & S A M>M I C\end{array}$

Fig. 6. High gamma SEEG activity and fMRI ROIs. Coronal view of the location of the SEEG sites and fMRI ROIs as in Fig. 2. Furthermore, the SEEG sites displaying a significant increase in high gamma band power after the SAM cue are coded in orange, whereas sites showing a stronger effect after the MIC cue are coded in blue.

line with a previous study by Huettel et al. (2004) who reported that the relation between changes in magnitude of the ERPs and the fMRI BOLD responses as a function of stimulus duration varies across cortical regions. The weak spatial colocalization among the two measures found in the present study and Huettel et al.'s study suggests that intracranial evoked responses are not informative about the hemodynamic responses when complex stimuli or behavioral tasks are considered.

Likewise, changes in the SEEG spectrum in the beta band $(15-30 \mathrm{~Hz})$, assessed by means of wavelet analysis, were significantly different in three SEEG sites that were located in proximity to the fMRI ROIs. However, the beta band response showed a stronger decrease in power after the MIC cue independently of their proximity to either SAM or MIC-related fMRI ROIs. Furthermore, four SEEG sites, which were closely placed to the fMRI ROIs did not show significant differences between the SAM and MIC conditions in the $15-30 \mathrm{~Hz}$ frequency band. We conclude that, at least in the present experimental paradigm, the spatial extent of the beta band modulations does not colocalize with the fMRI effects of the interaction analysis (Simon et al., 2002). The reason for this discrepancy could be due to the type of responsiveness of the beta rhythm to sensory stimuli: beta range oscillations are widely observed in the sensorimotor cortex in relation to motor behaviors in both humans ( Salmelin and Hari, 1994; Pfurtscheller and Lopes da Silva, 1999; Brovelli et al., 2002; Salenius and Hari, 2003) and nonhuman primates (Rougeul et al., 1979; Murthy and Fetz, 1992; Sanes and Donoghue, 1993; MacKay and Mendonca, 1995; Brovelli et al., 2004). In particular, power suppression in the beta band, known as event-related desynchronization (ERD), has been observed during tasks activating the sensorimotor cortex using both EEG (Pfurtscheller, 1981) and electrocorticographic (ECoG) techniques (Arroyo et al., 1993; Toro et al., 1994, Crone et al., 1998a). Thus, we might have anticipated that beta band SEEG changes would have been mostly affected by the motor intention component of the task (i.e., MIC cue) rather than by spatial attention and memory demands. This could explain the lack of spatial colocalization with the fMRI ROIs in the present study.

High gamma frequency activity dissociates spatial attention/ memory from motor planning and parallels hemodynamic changes

The frequency component of the SEEG signal that differentially changed in relation to SAM and MIC cues in discrete sites of the premotor cortex ranged from 60 to $200 \mathrm{~Hz}$, the so-called high gamma frequency range. This result provides electrophysiological confirmation of the nonmotor functions of the human premotor cortex, as suggested by results from monkey neurophysiology and human neuroimaging (Boussaoud and Wise, 1993a,b; Corbetta et al., 1993; Coull and Nobre, 1998; Courtney et al., 1998; Simon et al., 2002). Furthermore, among the SEEG responses to the SAM and MIC cues, the high gamma activity showed the best spatial correlation with the fMRI effects. The high gamma responses of four SEEG sites (sites 1, 2, 4 and 5) paralleled the fMRI findings in terms of proximity to the ROIs and selectivity to either the SAM or MIC cue (Figs. 5 and 6). In fact, only one site (site 9) displayed a significant difference in mean power across conditions without being localized close to any fMRI ROI. However, a closer inspection of its mean waveforms (Fig. 5b) shows that the significant difference mainly occurs between the post-SAM activity and the one preceding the MIC cue. Thus, we could expect site 9 to be located close to one ROI defined by the comparison between the Rest and SAM condition in the fMRI study (Simon et al., 2002), instead of being close to the ROIs defined from the SAM vs. MIC comparison. In fact, site 9 was approximately $11 \mathrm{~mm}$ from one fMRI ROI in the left dorsal premotor cortex which was engaged in spatial attention/ memory with respect to Rest condition (Table 2 of Simon et al., 2002). Finally, no difference in the high gamma band response was found in four SEEG sites (sites 3, 6, 7 and 8) even though they were located close to fMRI ROIs. This result shows that the BOLD response and high gamma band oscillations do not display a one-to-one relation. However, the fact that all the SEEG sites revealing a significant difference in high gamma band power were closely placed to the corresponding fMRI ROI suggests that the high gamma effect is more focused than the BOLD related responses.

Our results support a previous study showing a coupling between the BOLD response and the LFPs in a frequency band peaking around $73 \pm 21 \mathrm{~Hz}$ in the monkey (Logothetis et al., 2001). The reason why in our study the differential SEEG responses correlate with the fMRI results only in the high gamma band remains unclear. However, it is interesting to note that the high gamma power profile of the SEEG responses resembles the poststimulus time histograms of single neurons activity recorded in the monkey premotor cortex (Boussaoud and Wise, 1993a,b). Indeed, at sites 1 and 2, SEEG responses characterized by a steep onset followed by a slower decay, have a similar profile than SAMrelated neurons. Conversely, SEEG sites 4 and 5, with a relatively 
slower and sustained response, surprisingly resemble the response of MIC-related neurons (Boussaoud and Wise, 1993a,b). No study has yet described the relation between high frequency LFPs and spike timing during spatial attention and motor planning, but previous results from Pesaran et al. (2002) demonstrated that the spiking and LFP activity in the $25-90 \mathrm{~Hz}$ frequency band can become coherent during working memory in the monkey parietal cortex. In humans, high gamma power ECoG responses following movements of different body parts were found to occur in a more discrete topographical pattern than the beta ERD phenomena. Furthermore, somatotopically defined regions on the basis of high gamma oscillations in the sensorimotor cortex were consistent with maps generated by cortical electrical stimulation (Crone et al., 1998b). Furthermore, another study on 4 epileptic patients performing a face detection task showed a good spatial colocalization between the increase of high gamma band SEEG activity and the results from the fMRI literature using different but comparable tasks (Lachaux et al., in press). Taken together, these results provide evidence for a relation between high-frequency SEEG oscillations, spiking activity and hemodynamic responses.

\section{Concluding remarks and limitations}

The present study was motivated by a previous work which identified the human cortical regions selective for spatial attention and memory processes and motor intention (Simon et al., 2002). Given the lack of temporal information in the fMRI study, we measured the time course of the electrical activity within the fMRI ROIs and exploited the multidimensionality of the SEEG signal to define the components that discriminate motor intention from spatial attention and memory. High gamma frequency SEEG oscillations were found to differently respond to SAM and MIC cues according to their location in the cortex. Thus, we strengthened the idea that the primate premotor cortex contributes both to motor and nonmotor processes and most importantly, we identified a strong similarity between the temporal dynamics of gamma activity and that of single neurons under the same task.

This study is an attempt to clarify the relation between neurophysiological and hemodynamic measures, which might help to determine the optimal strategy to combine EEG-MEG and fMRI data to study the spatiotemporal dynamics of brain activity. We cannot exclude that the patient's fMRI would differ from the group study, because it was not possible to acquire fMRI for practical reasons. However, it seems unlikely, because, among the large number of electrodes analyzed, all the SEEG sites dissociating between SAM and MIC cues were located close to the corresponding ROIs of the group fMRI study. Furthermore, although the relation between the high gamma oscillatory activity and the BOLD effect is indirect, we believe that the data support more direct evidence from combined fMRI and electrophysiology in the monkey. The results open new perspectives for the use of high gamma frequency oscillations as an additional measure of brain activity worth taking into account in presurgical and clinical practice. We are aware that a single case study has limitations. Of course, the findings would gain more significance if reproduced in other subjects. However, intracranial electrodes in epileptic patients rarely involve the premotor cortex. We thus chose to present this rather unique case, because we think the results are of great interest for both neuroscientists and clinicians.

\section{Acknowledgments}

We would like to thank Martine Meunier, from the INCM, CNRS, Marseille, France, for interesting discussions and suggestions on the manuscript. We are also grateful to the patient who accepted to participate to this study and Dr. L. Minotti, Valérie Balle, Patricia Boschetti, Carole Chatelard, Véronique Dorlin, Eliane Gamblin and Martine Juillard from the Epilepsy Unit, CHU Grenoble, France. AB was supported by the European community 5th framework project "I-Learning". JPL was supported by the Fyssen Foundation.

\section{References}

Arroyo, S., Lesser, R.P., Gordon, B., Uematsu, S., Jackson, D., Webber, R., 1993. Functional significance of the mu rhythm of human cortex: an electrophysiologic study with subdural electrodes. Electroencephalogr. Clin. Neurophysiol. 87, 76-87.

Boussaoud, D., 2001. Attention versus intention in the primate premotor cortex. Neuroimage 14, 40-45.

Boussaoud, D., Wise, S.P., 1993a. Primate frontal cortex: neuronal activity following attention versus intentional cues. Exp. Brain Res. 95, 15-27.

Boussaoud, D., Wise, S.P., 1993b. Primate frontal cortex: effects of stimulus and movement. Exp. Brain Res. 95, 28-40.

Brovelli, A., Battaglini, P.P., Naranjo, J.R., Budai, R., 2002. Medium-range oscillatory network and the $20-\mathrm{Hz}$ sensorimotor induced potential. Neuroimage 16, 130-141.

Brovelli, A., Ding, M., Ledberg, A., Chen, Y., Nakamura, R., Bressler, S.L., 2004. Beta oscillations in a large-scale sensorimotor cortical network: directional influences revealed by Granger causality. Proc. Natl. Acad. Sci. U. S. A. 101, 9849-9854.

Corbetta, M., Akbudak, E., Conturo, T.E., Snyder, A.Z., Ollinger, J.M., Druthy, H.A., Linenweber, M.R., Petersen, S.E., Raichle, M.E., Van Essen, D.C., Shulman, G.L., 1993. Primate frontal cortex: neuronal activity following attention versus intentional cues. Exp. Brain Res. 95, $15-27$.

Coull, J.T., Nobre, A.C., 1998. Where and when to pay attention: the neural systems for directing attention to spatial locations and to time intervals as revealed by both PET and fMRI. J. Neurosci. 18, 7426-7435.

Courtney, S.M., Petit, L., Maisog, J.M., Ungerleider, L.G., Haxby, J.V., 1998. An area specialized for spatial working memory in human frontal cortex. Science 279, 1347-1351.

Crone, N.E., Miglioretti, D.L., Gordon, B., Sieracki, J.M., Wilson, M.T., Uematsu, S., Lesser, R.P., 1998a. Functional mapping of human sensorimotor cortex with electrocorticographic spectral analysis: I. Alpha and beta event-related desynchronization. Brain 121, $2271-2299$.

Crone, N.E., Miglioretti, D.L., Gordon, B., Lesser, R.P., 1998b. Functional mapping of human sensorimotor cortex with electrocorticographic spectral analysis: II. Event-related synchronization in the gamma band. Brain 121, 2301-2315

Devor, A., Dunn, A.K., Andermann, M.L., Ulbert, I., Boas, D.A., Dale, A.M., 2003. Coupling of total hemoglobin concentration, oxygenation, and neural activity in rat somatosensory cortex. Neuron.

Huettel, S.A., McKeown, M.J., Song, A.W., Hart, S., Spencer, D.D., Allison, T., McCarthy, G., 2004. Linking hemodynamic and electrophysiological measures of brain activity: evidence from functional MRI and intracranial field potentials. Cereb. Cortex 14, 165-173.

Kim, D.S., Ronen, I., Olman, C., Kim, S.G., Ugurbil, K., Toth, L.J., 2004. Spatial relationship between neuronal activity and BOLD functional MRI. Neuroimage 21, 876-885.

Lachaux, J.P., Rudrauf, D., Kahane, P., 2003. Intracranial EEG and human brain mapping. J. Physiol. (Paris) 97, 613-628.

Lachaux, J.P., Georgea, N., Tallon-Baudry, C., Martinerie, J., Hugueville, 
L., Minotti, L., Kahane, P., Renault, B., in press. The many faces of the gamma band response to complex visual stimuli. Neuroimage.

Lauritzen, M., Gold, L., 2003. Brain function and neurophysiological correlates of signals used in functional neuroimaging. J. Neurosci. 23, $3972-3980$.

Lebedev, M.A., Wise, S.P., 2001. Tuning for the orientation of spatial attention in dorsal premotor cortex. Eur. J. Neurosci. 13, 1002-1008.

Logothetis, N.K., Wandell, B.A., 2004. Interpreting the BOLD signal. Annu. Rev. Physiol. 66, 735-769.

Logothetis, N.K., Pauls, J., Augath, M., Trinath, T., Oeltermann, A., 2001. Neurophysiological investigation of the basis of the fMRI signal. Nature $412,150-157$.

MacKay, W.A., Mendonca, A.J., 1995. Field potential oscillatory bursts in parietal cortex before and during reach. Brain Res. 704, 167-174.

Murthy, V.N., Fetz, E.E., 1992. Coherent 25- to 35-Hz oscillations in the sensorimotor cortex of awake behaving monkeys. Proc. Natl. Acad. Sci. U. S. A. $89,5670-5674$.

Nemoto, M., Sheth, S., Guiou, M., Pouratian, N., Chen, J.W., Toga, A.W., 2004. Functional signal- and paradigm-dependent linear relationships between synaptic activity and hemodynamic responses in rat somatosensory cortex. J. Neurosci. 24, 3850-3861.

Pesaran, B., Pezaris, J.S., Sahani, M., Mitra, P.P., Andersen, R.A., 2002. Temporal structure in neuronal activity during working memory in macaque parietal cortex. Nat. Neurosci. 8, 805-811.

Pfurtscheller, G., 1981. Central beta rhythm during sensorimotor activities in man. Electroencephalogr. Clin. Neurophysiol. 51, 253-264.

Pfurtscheller, G., Lopes da Silva, F.H., 1999. Event-related EEG/MEG synchronization and desynchronization: basic principles. Clin. Neurophysiol. 110, 1842-1857.
Rougeul, A., Bouyer, J.J., Dedet, L., Debray, O., 1979. Fast somatoparietal rhythms during combined focal attention and immobility in baboon and squirrel monkey. Electroenceph. Clin. Neurophysiol. 46, $310-319$.

Salenius, S., Hari, R., 2003. Synchronous cortical oscillatory activity during motor action. Curr. Opin. Neurobiol. 13, 678-684.

Salmelin, R., Hari, R., 1994. Spatiotemporal characteristics of sensorimotor neuromagnetic rhythms related to thumb movement. Neuroscience 60 , $537-550$.

Sanes, J.N., Donoghue, J.P., 1993. Oscillations in local field potentials of the primate motor cortex during voluntary movement. Proc. Natl. Acad. Sci. U. S. A. $90,4470-4474$.

Sheth, S.A., Nemoto, M., Guiou, M., Walker, M., Pouratian, N., Toga, A.W., 2004. Linear and nonlinear relationships between neuronal activity, oxygen metabolism, and hemodynamic responses. Neuron 42 , $347-355$.

Simon, S.R., Meunier, M., Piettre, L., Berardi, A.M., Segebarth, C.M., Boussaoud, D., 2002. Spatial attention and memory versus motor preparation: premotor cortex involvement as revealed by fMRI. J. Neurophysiol. 88, 2047-2057.

Tallon-Baudry, C., Bertrand, O., 1999. Oscillatory gamma activity in humans and its role in object representation. Trends Cogn. Sci. 3, 151-162.

Toro, C., Deuschl, G., Thatcher, R., Sato, S., Kufta, C., Hallett, M., 1994. Event-related desynchronization and movement-related cortical potentials on the ECoG and EEG. Electroencephalogr. Clin. Neurophysiol. 93, 380-389.

Wise, S.P., Boussaoud, D., Johnson, P.B., Caminiti, R., 1997. Premotor and parietal cortex: corticocortical connectivity and combinatorial computations. Annu. Rev. Neurosci. 20, 25-42. 